Department of Pedintrics, Division of Medical Genetics, Emory University School of Medicine, Atlanta, Georgia

Address correspondence to: Louis J. Elsas II, MD, Division of Medical Genetics, Department of Pediatrics, Emory University School of Medicine, 2040 Ridgewood Drive, Atlanta, GA 30322. E-mail: lje@rw.ped.emory.edu 01998 Gervetics in Medicine. All rights reserved. $1098-3600 \$ 0.00$

\title{
The molecular biology of galactosemia
}

\author{
Louis J. Elsas II, MD, and Kent Lai, PhD
}

Classic galactosemia is an autosomal recessive disorder caused by the deficiency of galactose 1-phosphate uridyltransferase (GALT). Although the potentially lethal, neonatal hepatotoxic syndrome is prevented by newborn screening and galactose restriction, long-term outcome for older patients with galactosemia remains problematic. After the cloning and sequencing of the GALT gene, more than 130 mutations in the GALT gene have been associated with GALT deficiency; this review relates them to function and clinical outcome. Two common mutations, Q188R and $\mathrm{K} 285 \mathrm{~N}$, account for more than $70 \%$ of $\mathrm{G}$ alleles in the white population and are associated with classic galactosemia and impaired GALT function. In the black population, S135L accounts for $62 \%$ of the alleles causing galactosemia and is associated with good outcomes. A large $5 \mathrm{~kb}$ deletion in the GALT gene is found in Ashkenazim Jews. The Duarte galactosemia variant is caused by N314D. Homozygosity for N314D reduces GALT activity to $50 \%$. When either E203K or a 1721C $\rightarrow T$ transition (Los Angeles variant) are present in cis with N314D, GALT activity reverts to normal. In this review, we discuss the structural biology of these mutations as they affect both the GALT enzyme and patient outcome.

Key words: Galactosemia, galactose 1-phosphate uridyltransferase, mutations, outcome, ethnicity

\section{INTRODUCTION}

The conversion of galactose to glucose 1-phosphate is highly conserved throughout evolution and involves unique uridyltransfer through the enzyme galactose 1-phosphate uridyltransferase (GALT) (EC 2.7.7.12). Deficiency of GALT in humans was described in 1935 as the syndrome, galactosemia. ${ }^{1}$ Evaluation of outcome in patients with galactosemia began in the 1980s and an enigma was revealed by $1990 .^{2-5}$ Although neonates were screened and treated within days of birth by a galactose-restricted diet, older children had an unexpected poor outcome. Dysfunctions included ovarian failure, ${ }^{6}$ verbal dyspraxia, ${ }^{7}$ growth and developmental delays, ${ }^{8}$ and neurological signs of cortical and extrapyramidal tract impairment. ${ }^{9}$ Because GALT enzyme structure is conserved through evolution, the human GALT complimentary-DNA (cDNA) could be cloned using probes homologous to known bacterial amino acid sequences. When the cDNA and the gene for GALT were cloned and sequenced, ${ }^{10-13}$ the first mutations in the GALT gene were associated with human galactosemia. ${ }^{14-19} \mathrm{By}$ 1990 , genotype/phenotype relationships were reported. ${ }^{20}$ Because the human GALT gene has $46 \%$ identity with the amino acid sequence of Escherichia coli and $100 \%$ in defined catalytic regions, ${ }^{11-12}$ considerable progress was made in understanding the mechanisms by which mutations altered enzyme function. The normal GALT enzyme exhibits pingpong, bi-bi kinetics ${ }^{21-23}$ which denotes that one domain of the enzyme binds uridine diphosphate (UDP)- glucose to form a GALT enzyme-UDPglucose intermediate. Glucose 1-phosphate is released whereas uridine monophosphate remains bound to GALT. Galactose 1-phosphate then binds with the GALT-uridine monophosphate complex to form GALT-UDP-galactose, UDP-galactose is released, and GALT is freed for the next round of reactions (Fig. 1). Using the E. coli GALT protein crystal, ${ }^{24-25}$ specific structures for these unique reactions could be deduced for the human GALT. One site of particular interest is histidine-proline-histidine-cysteine-glutamine at amino acids 184 to 188 in the human GALT protein. This important site resides on a $\beta$-sheet at the core of the catalytic site as revealed in its three-dimensional crystal structure. The critical role of histidine at position 186 in catalysis was confirmed from experiments of sitedirected mutagenesis in the equivalent $E$. coli GALT protein. ${ }^{24}$ Because the most common $G$ allele in the white population is an arginine substituted for a glutamine at position $188(\mathrm{Q} 188 \mathrm{R})$, the role of glutamine in these catalytic reactions is of considerable interest. Other natural mutations that ablate, partially impair, salvage, or increase GALT activity were reported and are clarifying the normal structural biochemistry of human GALT.

It is thus timely and clinically important to review mutations in the human GALT gene that cause galactosemia. These mutations are summarized and tabulated (Table 1) with specific reference to their prevalence in ethnic groups included in Table 2 . The methods used to validate GALT variations as causative of galactosemia, and the relationship of these mutations to long-term outcome are discussed.

\section{STIUGTURAL BCLOGY OF COMMON MISSzISE MUTATONS}

The Q188R mutation was first reported in 1991 and is the most common mutation among classic galactosemics of white ethnicity occurring with an allele frequency of $70 \% .^{12,14}$ When this mutation was expressed initially in monkey COS cells, it yielded $\approx 10 \%$ of the wild-type GALT control activity. ${ }^{14}$ This incorrect observation was caused by endogenous GALT present in the monkey COS cells. The same mutation has no activity in yeast and $E$. coli expression systems in which endogenous GALT is absent. ${ }^{26,27}$ 


\begin{tabular}{|c|c|c|c|c|c|}
\hline & & & Nucleotide chan & human GALT gene & \\
\hline Amino Acid Change & Exon & Nucleotide Change & Conserved amino acid & In vitro expression analysis & Reference \\
\hline (I) Missense "Mutations" & & & & & \\
\hline $\mathrm{Asp}^{28} \mathrm{Tyr}(\mathrm{D} 28 \mathrm{Y})$ & I & $\underline{\mathrm{GAC}} \rightarrow \underline{\mathrm{TAC}}$ & No & Not done & Greber-Platzer et al., 1995 (per. comm.) \\
\hline $\mathrm{Ile}^{32} \mathrm{Asn}(\mathrm{I} 32 \mathrm{~N})$ & II & $\mathrm{ATC} \rightarrow \mathrm{AAC}$ & No & Not done & Greber-Platzer et al., 1995 (per. comm.) \\
\hline $\mathrm{Gln}^{38}$ Pro (Q38P) & II & $\mathrm{CA} G \rightarrow \mathrm{C} \underline{\mathrm{C} G}$ & No & Not done & Greber-Platzer et al., 1995 (per. comm.) \\
\hline $\mathrm{Val}^{14}$ Leu (V44L) & II & $\underline{\mathrm{GTG}} \rightarrow \underline{\mathrm{ITG}}$ & YES & Not done & This comm. \\
\hline $\mathrm{Val}^{44}$ Met (V44M) & II & $\underline{\mathrm{GTG}} \rightarrow \underline{\mathrm{ATG}}$ & YES & COS cells & 15 \\
\hline $\operatorname{Arg}^{51} \operatorname{Leu}^{b}(\mathrm{R} 51 \mathrm{~L})$ & II & $\mathrm{CG} C \rightarrow \mathrm{CIC}$ & NO & Not done & 57 \\
\hline $\mathrm{Gly}^{55} \mathrm{Cys}$ (G55C) & II & $\underline{G G T} \rightarrow \underline{T G T}$ & No & Not done & Tyfield, per. comm.e \\
\hline Leu $^{62}$ Met (L62M) & II & $\underline{\mathrm{CTG}} \rightarrow \underline{\mathrm{ATT}}$ & No & COS cells & 15 \\
\hline $\operatorname{Arg}^{67} \mathrm{Cys}^{b}(\mathrm{R} 67 \mathrm{C})$ & II & $\underline{\mathrm{CGC}} \rightarrow \underline{\mathrm{TGC}}$ & No & Not done & 53 \\
\hline Leu $^{74}$ Pro (L74P) & II & $\mathrm{CTG} \rightarrow \mathrm{CCG}$ & YES & COS cells & 17 \\
\hline $\mathrm{Ala}^{81} \mathrm{Thr}(\mathrm{A81T})$ & II & $\underline{\mathrm{GCC}} \rightarrow \underline{\mathrm{ACC}}$ & NO & Not done & 12 \\
\hline Asn"7 Ser (N97S) & III & $\mathrm{A} \underline{\mathrm{AG}} \rightarrow \mathrm{A} \underline{\mathrm{G}} \mathrm{C}$ & YES & Not done & 48 \\
\hline $\mathrm{Asp}^{98} \mathrm{Asn}(\mathrm{D} 98 \mathrm{~N})$ & III & $\underline{\mathrm{GAC}} \rightarrow \underline{\mathrm{AAC}}$ & YES & Not done & Tyfield, per. comm.e \\
\hline $\operatorname{Asp}^{113}$ Asn (D113N) & IV & $\underline{\mathrm{GAT}} \rightarrow \underline{\mathrm{AAT}}$ & No & Not done & This comm. \\
\hline His $^{114}$ Leu (H114L) & IV & $\mathrm{CAT} \rightarrow \mathrm{CTT}$ & No & Not done & This comm. \\
\hline Phe ${ }^{117}$ Ser (F117S) & IV & $\mathrm{T} \underline{\mathrm{TC}} \rightarrow \mathrm{T} \underline{\mathrm{C}} \mathrm{C}$ & No & Not done & This comm. \\
\hline $\mathrm{Gln}^{118} \mathrm{His}(\mathrm{Q} 118 \mathrm{H})$ & IV & $\mathrm{CA} \underline{A} \rightarrow \mathrm{CA} \underline{C}$ & No & Not done & This comm. \\
\hline $\operatorname{Arg}^{123} \mathrm{Gly}$ (R123G) & IV & $\underline{\mathrm{CGA}} \rightarrow \underline{\mathrm{GGA}}$ & YES & Not done & Greber-Platzer et al., 1995 (per. comm.) \\
\hline $\operatorname{Arg}^{123} G \ln (R 123 Q)$ & IV & $\mathrm{C} \underline{\mathrm{GA}} \rightarrow \mathrm{C} \underline{\mathrm{A} A}$ & YES & Not done & This comm. \\
\hline $\mathrm{Val}^{125} \mathrm{Ala}(\mathrm{V} 125 \mathrm{~A})$ & IV & $\mathrm{GTC} \rightarrow \mathrm{G} \underline{\mathrm{CC}}$ & No & Not done & This comm. \\
\hline Lys $^{127}$ Glu (K127E) & V & $\underline{\mathrm{AAG}} \rightarrow \underline{\mathrm{GAG}}$ & NO & Not done & This comm. \\
\hline $\mathrm{Cys}^{130} \operatorname{Tyr}(\mathrm{Cl} 130 \mathrm{Y})$ & $\mathrm{V}$ & $\mathrm{TGC} \rightarrow \mathrm{TAC}$ & YES & Not done & This comm. \\
\hline $\mathrm{His}^{132} \mathrm{Tyr}(\mathrm{H} 132 \mathrm{Y})$ & $\mathrm{V}$ & $\underline{\mathrm{CAC}} \rightarrow \underline{\mathrm{TAC}}$ & NO & Not done & This comm. \\
\hline $\operatorname{Ser}^{135} \operatorname{Leu}^{b, c}(\mathrm{~S} 135 \mathrm{~L})$ & $\mathrm{v}$ & $\mathrm{T} \underline{\mathrm{CG}} \rightarrow \mathrm{T} \underline{\mathrm{TG}}$ & NO & COS cells, gal7 yeasts, gal- E. coli & 17 \\
\hline $\operatorname{Thr}^{138} \operatorname{Met}^{b}(\mathrm{~T} 138 \mathrm{M})$ & $\mathrm{V}$ & $\mathrm{A} \underline{\mathrm{C} G} \rightarrow \mathrm{A} \underline{\underline{T}} \mathrm{G}$ & YES & Not done & 52,58 \\
\hline Leu $^{139}$ Pro (L139P ) & $\mathrm{V}$ & $\mathrm{CTG} \rightarrow \mathrm{CC} G$ & NO & Not done & 12 \\
\hline $\mathrm{Met}^{142} \mathrm{Val}(\mathrm{M} 142 \mathrm{~V})$ & $\mathrm{V}$ & $\mathrm{ATG} \rightarrow \mathrm{GTG}$ & NO & COS cells & 48 \\
\hline $\mathrm{Met}^{142}$ Lys (M142K) & $\mathrm{V}$ & $\mathrm{ATG} \rightarrow \mathrm{A} \underline{\underline{A}}$ & NO & COS cells & 15 \\
\hline $\operatorname{Ser}^{143} \operatorname{Leu}^{b}(S 143 \mathrm{~L})$ & $\mathrm{V}$ & $\mathrm{TCG} \rightarrow \mathrm{TTG}$ & NO & Not done & Greber-Platzer et al., 1995 (per. comm.) \\
\hline $\operatorname{Arg}^{148} \operatorname{Trp}^{b}(\mathrm{R} 148 \mathrm{~W})$ & $\mathrm{v}$ & $\underline{\mathrm{CGG}} \rightarrow \underline{\mathrm{TGG}}$ & NO & Cos cells & 16 \\
\hline $\operatorname{Arg}^{148} \mathrm{Gln}^{b}(\mathrm{R} 148 \mathrm{Q})$ & $\mathrm{V}$ & $\mathrm{CGG} \rightarrow \mathrm{C} \underline{\mathrm{A} G}$ & NO & Not done & 58 \\
\hline $\operatorname{Arg}^{148} \mathrm{Gly}^{b}(\mathrm{R} 148 \mathrm{G})$ & $\mathrm{V}$ & $\underline{\mathrm{C} G G} \rightarrow \underline{\mathrm{GG}}$ & NO & Not done & This comm. \\
\hline $\mathrm{Val}^{150} \mathrm{Leu}(\mathrm{V} 150 \mathrm{~L})$ & $\mathrm{V}$ & $\underline{\mathrm{GTT}} \rightarrow \underline{\mathrm{CTT}}$ & NO & Not done & 49 \\
\hline $\mathrm{Val}^{151} \mathrm{Ala}(\mathrm{V} 151 \mathrm{~A})$ & $\mathrm{v}$ & $\mathrm{GTT} \rightarrow \mathrm{G} \underline{\mathrm{CT}}$ & YES & gal7 yeasts & 58 \\
\hline $\operatorname{Trp}^{154}$ Gly (W154G) & $\mathrm{V}$ & $\underline{T G G} \rightarrow \underline{G G G}$ & YES & Not done & This comm. \\
\hline
\end{tabular}




\begin{tabular}{|c|c|c|c|c|c|}
\hline Amino Acid Change & Exon & Nucleotide Change & Conserved amino acid & In vitro expression analysis & Reference \\
\hline $\mathrm{Phe}^{171} \operatorname{Ser}(\mathrm{F} 171 \mathrm{~S})$ & VI & $\mathrm{T} \underline{\mathrm{TT}} \rightarrow \mathrm{T} \underline{\mathrm{CT}}$ & YES & $\cos$ cells & 17 \\
\hline Gly ${ }^{179}$ Asp (G179D) & VI & $\mathrm{GCC} \rightarrow \mathrm{GAC}$ & YES & COS cells & 48 \\
\hline $\operatorname{Pro}^{183} \mathrm{Thr}(\mathrm{P} 183 \mathrm{~T})$ & VI & $\underline{\mathrm{C} C C} \rightarrow \underline{\mathrm{ACC}}$ & YES & Not done & 49 \\
\hline $\mathrm{His}^{184} \mathrm{Gln}(\mathrm{H} 184 \mathrm{Q})$ & VI & $\mathrm{CA} \underline{\mathrm{C}} \rightarrow \mathrm{CA} \underline{\mathrm{A}}$ & YES & Not done & This comm. \\
\hline $\mathrm{Gln}^{188} \operatorname{Arg}(\mathrm{Q} 188 \mathrm{R})$ & VI & $\mathrm{C} \underline{\mathrm{A} G} \rightarrow \mathrm{C} \underline{\mathrm{G}} \mathrm{G}$ & YES & COS cells, gal7 yeasts, gal-E. coli & 14 \\
\hline $\operatorname{Ser}^{192}$ Asn (S192N) & VII & $\mathrm{AGC} \rightarrow \mathrm{AAC}$ & NO & Not done & 58 \\
\hline Phe ${ }^{194}$ Leu (F194L) & VII & $\underline{\mathrm{TTC}} \rightarrow \underline{\mathrm{CTC}}$ & NO & Not done & Tyfield, per. comm. ${ }^{e}$ \\
\hline Leu $^{195}$ Pro (L195P) & VII & $\mathrm{CT} G \rightarrow \mathrm{C} \underline{\mathrm{CG}}$ & NO & COS cells & 16 \\
\hline $\mathrm{Ile}^{198} \mathrm{Met}(\mathrm{I198M})$ & VII & $\mathrm{ATT} \rightarrow \mathrm{AT} \underline{\mathrm{G}}$ & NO & Not done & This comm. \\
\hline $\mathrm{Ile}^{198} \operatorname{Thr}(\mathrm{I} 198 \mathrm{~T})$ & VII & $\mathrm{ATT} \rightarrow \mathrm{A} \underline{\mathrm{CT}}$ & NO & Not done & This comm. \\
\hline $\mathrm{Ala}^{199} \mathrm{Thr}(\mathrm{A} 199 \mathrm{~T})$ & VII & $\underline{\mathrm{GCC}} \rightarrow \underline{\mathrm{ACC}}$ & NO & Not done & 48 \\
\hline $\operatorname{Arg}^{201} \mathrm{His}^{b}(\mathrm{R} 201 \mathrm{H})$ & VII & $\mathrm{CG} T \rightarrow \mathrm{C} \underline{\mathrm{A} T}$ & NO & Not done & 58 \\
\hline $\mathrm{Glu}^{203}$ Lys (E203K) & VII & $\underline{\mathrm{G} A G} \rightarrow \underline{\mathrm{A} A G}$ & NO & gal7 yeasts & 58 \\
\hline $\mathrm{Tyr}^{209}$ Cys (Y209C) & VII & $\mathrm{T} \underline{\mathrm{AT}} \rightarrow \mathrm{T} \underline{\mathrm{GT}}$ & YES & gal-E. coli & This comm. \\
\hline $\mathrm{Tyr}^{209} \operatorname{Ser}(\mathrm{Y} 209 \mathrm{~S})$ & VII & $\mathrm{T} \underline{\mathrm{A} T} \rightarrow \mathrm{T} \underline{\mathrm{C}} \mathrm{T}$ & YES & Not done & Kozak, per. comm \\
\hline $\mathrm{Gln}^{212} \mathrm{His}(\mathrm{Q} 212 \mathrm{H})$ & VII & $\mathrm{C} \underline{\mathrm{TG}} \rightarrow \mathrm{C} \underline{\mathrm{CG}}$ & NO & Not done & Tyfield, per. comm. \\
\hline Leu $^{217}$ Pro (L217P) & VII & $\mathrm{CT} A \rightarrow \mathrm{C} \underline{C A}$ & NO & Not done & This comm. \\
\hline Leu $^{226}$ Pro (L226P) & VII & $\mathrm{C} \underline{T A} \rightarrow \mathrm{C} \underline{\mathrm{CA}}$ & NO & Not done & This comm. \\
\hline $\operatorname{Arg}^{231} \mathrm{His}(\mathrm{R} 231 \mathrm{H})$ & VIII & $\mathrm{CG} T \rightarrow \mathrm{CA} T$ & YES & COS cells & 47 \\
\hline $\operatorname{Trp}^{249} \operatorname{Arg}(\mathrm{W} 249 \mathrm{R})$ & VIII & $\underline{T} G G \rightarrow \underline{A G G}$ & YES & COS cells & 48 \\
\hline Tyrr ${ }^{251}$ Cys (Y251C) & VIII & $\mathrm{T} \underline{\mathrm{A}} \mathrm{C} \rightarrow \mathrm{T} \underline{\mathrm{G}} \mathrm{C}$ & NO & Not done & Tyfield, per. comm. \\
\hline $\operatorname{Tyr}^{251} \operatorname{Ser}(Y 251 S)$ & VIII & $\mathrm{T} \underline{\mathrm{AC}} \rightarrow \mathrm{T} \underline{C} \mathrm{C}$ & NO & Not done & This comm. \\
\hline $\operatorname{Arg}^{258} \mathrm{Cys}^{b}(\mathrm{R} 258 \mathrm{C})$ & VIII & $\underline{\mathrm{CGT}} \rightarrow \underline{\mathrm{TGT}}$ & NO & gal2 E. coli & This comm. \\
\hline $\operatorname{Arg}^{259} \operatorname{Trp}^{b}(\mathrm{R} 259 \mathrm{~W})$ & VIII & $\underline{\mathrm{C} G G} \rightarrow \underline{\mathrm{TGG}}$ & NO & Not done & 52 \\
\hline $\mathrm{Arg}^{262} \mathrm{Pro}(\mathrm{R} 262 \mathrm{P})$ & VIII & $\mathrm{C} \underline{G G} \rightarrow \mathrm{C} \underline{C G}$ & NO & Not done & This comm. \\
\hline $\operatorname{Arg}^{273}$ Gly (R273GF) & VIII & $\underline{\mathrm{CGT}} \rightarrow \underline{\mathrm{GGT}}$ & NO & Not done & Tyfield, per. comm. e $^{2}$ \\
\hline $\mathrm{Leu}^{282} \mathrm{Val}(\mathrm{L} 282 \mathrm{~V})$ & IX & $\underline{\mathrm{CTC}} \rightarrow \underline{\mathrm{GTC}}$ & YES & Not done & This comm. \\
\hline Lys $^{285}$ Asn (K285N) & IX & $\mathrm{AAG} \rightarrow \mathrm{AAT}$ & No & gal-E. coli & 12 \\
\hline $\mathrm{Leu}^{289} \mathrm{Arg}$ (L289R) & IX & $\mathrm{CTC} \rightarrow \mathrm{CG} \mathrm{C}$ & YES & Not done & This comm. \\
\hline $\mathrm{Glu}^{291}$ Lys (E291K) & IX & $\underline{\mathrm{G} A G} \rightarrow \underline{\mathrm{AAG}}$ & NO & Not done & 58 \\
\hline Glu $^{308}$ Lys (E308K) & IX & $\underline{\mathrm{G} A G} \rightarrow \underline{\mathrm{A} A G}$ & NO & Not done & 58 \\
\hline $\mathrm{Asn}^{314} \mathrm{Asp}^{c, d}(\mathrm{~N} 314 \mathrm{D})$ & $\mathrm{x}$ & $\underline{\mathrm{A} A C} \rightarrow \underline{\mathrm{GAC}}$ & NO & COS cells, gal7 yeasts, gal- E. coli & 15 \\
\hline $\mathrm{Gln}^{317} \operatorname{Arg}(\mathrm{Q} 317 \mathrm{R})$ & $\mathbf{X}$ & $\mathrm{CAG} \rightarrow \mathrm{CGG}$ & NO & Not done & Tyfield, per. comm. $^{e}$ \\
\hline $\mathrm{Gln}^{317} \mathrm{His}(\mathrm{Q} 317 \mathrm{H})$ & $\mathrm{X}$ & $\mathrm{CAG} \rightarrow \mathrm{CAT}$ & NO & Not done & This comm. \\
\hline $\mathrm{His}^{319} \mathrm{Gln}(\mathrm{H} 319 \mathrm{Q})$ & $\mathrm{X}$ & $\mathrm{CA} \underline{\mathrm{C}} \rightarrow \mathrm{CA} \underline{\mathrm{A}}$ & YES & COS cells & 18 \\
\hline $\mathrm{Ala}^{320} \mathrm{Thr}(\mathrm{A} 320 \mathrm{~T})$ & $\mathbf{x}$ & $\underline{\mathrm{GCT}} \rightarrow \underline{\mathrm{ACT}}$ & NO & Not done & 58 \\
\hline
\end{tabular}




\begin{tabular}{|c|c|c|c|c|c|}
\hline Amino Acid Change & Exon & Nucleotide Change & Conserved amino acid & In vitro expression analysis & Reference \\
\hline $\mathrm{Tyr}^{323} \mathrm{His}(\mathrm{Y} 323 \mathrm{H})$ & $\mathrm{X}$ & $\mathrm{IAC} \rightarrow \underline{\mathrm{C} A C}$ & YES & Not done & Tyfield, per. comm. ${ }^{e}$ \\
\hline $\mathrm{Tyr}^{323}$ Asp (Y323D) & $\mathbf{X}$ & $\underline{T A C} \rightarrow \underline{G A C}$ & YES & Not done & 58 \\
\hline $\mathrm{Pro}^{324} \operatorname{Ser}(\mathrm{P} 324 \mathrm{~S})$ & $\mathrm{X}$ & $\underline{\mathrm{CCT}} \rightarrow \underline{\mathrm{TCT}}$ & YES & Not done & Tyfield and Boleda, 1995 (per. comm.) \\
\hline $\operatorname{Pro}^{325} \operatorname{Leu}^{b}(\mathrm{P} 325 \mathrm{~L})$ & $\mathrm{X}$ & $\mathrm{CCG} \rightarrow \mathrm{CTG}$ & YES & Not done & This comm. \\
\hline $\mathrm{Arg}^{328} \mathrm{His}(\mathrm{R} 328 \mathrm{H})$ & $\mathbf{X}$ & $\mathrm{CG} \rightarrow \mathrm{C} \rightarrow \underline{A C}$ & YES & Not done & This comm. \\
\hline $\mathrm{Ser}^{329}$ Phe (S329F) & $\mathbf{X}$ & $\mathrm{T} \underline{\mathrm{CT}} \rightarrow \mathrm{T} \underline{T} \mathrm{~T}$ & YES & Not done & Tyfield, per. comm. ${ }^{e}$ \\
\hline $\mathrm{Ala}^{330} \mathrm{Val}(\mathrm{A} 330 \mathrm{~V})$ & $\mathbf{x}$ & $\mathrm{G} \underline{\mathrm{CC}} \rightarrow \mathrm{GIC}$ & YES & Not done & 53 \\
\hline $\operatorname{Arg}^{333} \operatorname{TrP}^{b}(\mathrm{R} 333 \mathrm{~W})$ & $\mathrm{x}$ & $\underline{\mathrm{C} G G} \rightarrow \underline{\mathrm{TGG}}$ & YES & COS cells & 14 \\
\hline $\operatorname{Arg}^{333} \mathrm{Gly}^{b}(\mathrm{R} 333 \mathrm{G})$ & $\mathrm{X}$ & $\underline{\mathrm{C} G G} \rightarrow \underline{\mathrm{GGG}}$ & YES & Not done & 12 \\
\hline $\operatorname{Arg}^{333} \operatorname{Gln}^{b}(\mathrm{R} 333 \mathrm{Q})$ & $\mathrm{X}$ & $\mathrm{CGG} \rightarrow \mathrm{CA} G$ & YES & Not done & 48 \\
\hline $\mathrm{Lys}^{334} \mathrm{Arg}(\mathrm{K} 334 \mathrm{R})$ & $\mathrm{X}$ & $\mathrm{A} \underline{A A} \rightarrow \mathrm{A} \underline{G} A$ & YES & Not done & 12 \\
\hline $\mathrm{Met}^{336} \mathrm{Leu}(\mathrm{M} 336 \mathrm{~L})$ & $\mathbf{X}$ & $\underline{\mathrm{ATG}} \rightarrow \underline{\mathrm{TTG}}$ & NO & Not done & This comm. \\
\hline $\mathrm{Gln}^{344}$ Lys (Q344K) & $\mathbf{X}$ & $\underline{\mathrm{C}} \mathrm{AG} \rightarrow \underline{\mathrm{AAG}}$ & NO & Not done & This comm. \\
\hline $\mathrm{Thr}^{350} \mathrm{Ala}(\mathrm{T} 350 \mathrm{~A})$ & $\mathrm{X}$ & $\underline{\mathrm{ACC}} \rightarrow \underline{\mathrm{GCC}}$ & NO & Not done & 52 \\
\hline \multicolumn{6}{|l|}{ (II) Nonsense "Mutations" } \\
\hline $\mathrm{Gln}^{54} \operatorname{Stop}(\mathrm{Q} 54 \mathrm{X})$ & II & $\underline{\mathrm{CAG}} \rightarrow \underline{\mathrm{TAG}}$ & NO & Not done & This comm. \\
\hline $\operatorname{Arg}^{80}$ Stop $^{*}(\mathrm{R} 80 \mathrm{X})$ & II & $\mathrm{CGA} \rightarrow \underline{\mathrm{TGA}}$ & YES & Not done & Tyfield, per. comm. $^{e}$ \\
\hline $\operatorname{Trp}^{154}$ Stop (W154X) & $\mathrm{V}$ & $\mathrm{TG} \underline{\mathrm{G}} \rightarrow \mathrm{TG} \underline{\mathrm{A}}$ & YES & Not done & This comm. \\
\hline $\operatorname{Arg}^{204}$ Stop $^{*}(\mathrm{R} 204 \mathrm{X})$ & VII & $\underline{\mathrm{CGA}} \rightarrow \underline{\mathrm{TGA}}$ & NO & Not done & Tyfield, per. comm. ${ }^{e}$ \\
\hline $\operatorname{Gln}^{212}$ Stop (Q212X) & VII & $\underline{\mathrm{C}} \mathrm{AG} \rightarrow \underline{\mathrm{TAG}}$ & NO & Not done & 54 \\
\hline $\operatorname{Trp}^{249}$ Stop (W249X) & VIII & $\mathrm{TGG} \rightarrow \mathrm{TG} \underline{\mathrm{A}}$ & YES & COS cells & 48 \\
\hline Leu $^{264}$ Stop (L264X) & VIII & $\underline{\mathrm{CTA}} \rightarrow \mathrm{TAG}$ & No & Not done & This comm. \\
\hline \multicolumn{6}{|c|}{$\Delta C$ (bp2164), insert $G$ (bp 2167 on same allele) } \\
\hline $\operatorname{Trp}^{316}$ Stop (W316X) & $\mathrm{X}$ & $\mathrm{TGG} \rightarrow \mathrm{T} \underline{A G}$ & NO & Not done & 53 \\
\hline Glu $^{340}$ Stop (E340X) & $\mathrm{X}$ & $\underline{\mathrm{GAA}} \rightarrow \underline{\mathrm{TAA}}$ & YES & Not done & 54 \\
\hline $\mathrm{Gln}^{353}$ Stop (Q353X) & $\mathrm{X}$ & $\underline{\mathrm{C} A G} \rightarrow \underline{\mathrm{TAG}}$ & YES & Not done & Tyrifle, per. comm.e \\
\hline Tyr ${ }^{366}$ Stop (Y366X) & $\mathrm{XI}$ & $\mathrm{TAC} \rightarrow \mathrm{TA} \underline{\mathrm{A}}$ & NO & Not done & Greber-Platzer et al., 1995 (per. comm.) \\
\hline $\mathrm{Gln}^{370}$ Stop (Q370X) & $\mathrm{XI}$ & $\underline{\mathrm{C} A G} \rightarrow \underline{\mathrm{TAG}}$ & NO & Not done & 19 \\
\hline \multicolumn{6}{|c|}{ (III) Small Insertions/Deletions } \\
\hline 528 ins $\mathrm{G}$ & II & insG(bp528) & NO & Not done & 49 \\
\hline S112fs insA & IV & $333 / 334$ ins $\mathrm{A}$ & NO & Not done & Tyfield, per. comm..$^{e}$ \\
\hline W134fs delT & $\mathrm{V}$ & 400 delT & NO & Not done & Tyfield, April 1997 \\
\hline$\Delta C($ bp1677) & VII & $\Delta C($ bp1677) & NO & Not done & This comm. \\
\hline L256/P257fs del & VIII & 768-770 delGCCfs & NO & Not done & Tyfield, per. comm. ${ }^{e}$ \\
\hline$\Delta$ amino acids $\$ 260-263$ & VIII & $\Delta$ amino acids $\# 260-263$ & NO & Not done & This comm. \\
\hline$\Delta \mathrm{T}(\mathrm{bp} 2359)$ & IX & $\Delta \mathrm{T}(\mathrm{bp} 2359)$ & YES & Not done & This comm. \\
\hline
\end{tabular}




\begin{tabular}{|c|c|c|c|c|c|}
\hline Amino Acid Change & Exon & Nucleotide Change & Conserved amino acid & In vitro expression analysis & Reference \\
\hline$\Delta \mathrm{C}(\mathrm{bp} 2756)$ & $\mathrm{X}$ & $\Delta \mathrm{C}(\mathrm{bp} 2756)$ & NO & Not done & This comm. \\
\hline$\Delta C($ bp2782) & $\mathrm{X}$ & $\Delta C($ bp2782) & YES & Not done & 48 \\
\hline L327delC & $\mathrm{X}$ & 979delCfs & YES & Not done & Tyfield, per. comm. ${ }^{e}$ \\
\hline $\begin{array}{l}\text { P351fsdelC } \\
\text { (IV) Gross Deletion }\end{array}$ & \multicolumn{4}{|c|}{ (IV) Gross Deletion } & Tyfield, per. comm..$^{e}$ \\
\hline Deletion (>5kb) & $1-x$ & - & - & Not done & 46 \\
\hline \multicolumn{6}{|c|}{ (V) Splice Site "Mutations" } \\
\hline $318 \mathrm{~A} \rightarrow \mathrm{G}$ & - & $\underline{A} \rightarrow \underline{G}(\mathrm{bp} 318)$ & - & Not done & 47 \\
\hline IVSC & - & $\underline{\mathrm{A}} \rightarrow \underline{\mathrm{C}}(\mathrm{bp} 956)$ & - & Not done & 58 \\
\hline IVS3 nt $+29 \mathrm{G} \rightarrow \mathrm{C}$ & - & $c .328+29 \mathrm{G} \rightarrow \mathrm{C}$ & 一 & Not done & Tyfield, per. comm. ${ }^{e}$ \\
\hline IVS4 nt+ l & - & c. $377+1 \mathrm{G} \rightarrow \mathrm{C}$ & - & Not done & 48 \\
\hline IVS4 nt $-27 \mathrm{G} \rightarrow \mathrm{C}$ & - & $c .378-27 \mathrm{G} \rightarrow \mathrm{C}$ & - & Not done & Tyfield, per. comm. ${ }^{e}$ \\
\hline IVS5 $\mathrm{nt}+62 \mathrm{G} \rightarrow \mathrm{A}$ & - & $c .507+62 \mathrm{G} \rightarrow \mathrm{A}$ & - & Not done & Tyfield, per. comm. ${ }^{e}$ \\
\hline IVS5 nt $-24 \mathrm{G} \rightarrow \mathrm{A}$ & - & c. $508-24 \mathrm{G} \rightarrow \mathrm{A}$ & - & Not done & Tyfield, per. comm. ${ }^{e}$ \\
\hline IVSF nt 1 & - & $\underline{G} \rightarrow \underline{A}($ bp 1472) & - & Not done & This comm. \\
\hline IVSF & - & $\underline{A} \rightarrow \underline{G}(\mathrm{bp} 1631)$ & - & Not done & 58 \\
\hline IVS7 nt +2 & - & c. $687+2 \mathrm{~T} \rightarrow \mathrm{C}$ & - & Not done & Tyfield, per. comm..$^{e}$ \\
\hline IVS8 $n t+13 \mathrm{~A} \rightarrow \mathrm{G}$ & - & $c .820+13 \mathrm{~A} \rightarrow \mathrm{G}$ & - & Not done & Tyfield, per. comm. ${ }^{e}$ \\
\hline IVS8 nt $+32 \mathrm{~A} \rightarrow \mathrm{G}$ & - & c. $820+32 \mathrm{~A} \rightarrow \mathrm{G}$ & - & Not done & Tyfield, per. comm. ${ }^{e}$ \\
\hline IVS8 nt $+58 \mathrm{G} \rightarrow \mathrm{T}$ & - & $c .820+58 \mathrm{G} \rightarrow \mathrm{T}$ & - & Not done & Tyfield, per. comm..$^{e}$ \\
\hline IVSH nt 13 & - & $\underline{\mathrm{A}} \rightarrow \underline{\mathrm{G}}(\mathrm{bp} 2207)$ & - & Not done & 48 \\
\hline IVSJ & - & $\underline{C} \rightarrow \mathrm{T}$ (bp2919) & - & Not done & 59 \\
\hline \multicolumn{6}{|c|}{ (VI) Selected Polymorphisms } \\
\hline SacI bp1391 & Intron & $\underline{G} \rightarrow A(b p 1391)$ & - & Not done & 42 \\
\hline Tyr 165 Tyr (Y165Y) & $\mathrm{v}$ & $\mathrm{TAC} \rightarrow \mathrm{TAT}$ & NO & Not done & Tyfield, per. comm.e \\
\hline Leu 195 Leu (L195L) & VII & $\underline{\mathrm{CTG}} \rightarrow \underline{\mathrm{TTG}}$ & NO & Not done & Tyfield, per. comm. ${ }^{e}$ \\
\hline Leu 218 Leu (L218L) & VII & $\underline{\mathrm{CTA}} \rightarrow \underline{\mathrm{TTA}}$ & NO & Not done & 44 \\
\hline Gly 302 Gly (G302G) & IX & $\mathrm{GGG} \rightarrow \mathrm{GG} \underline{\mathrm{A}}$ & NO & Not done & This comm. \\
\hline His 315 His $(\mathrm{H} 315 \mathrm{H})$ & $\mathrm{X}$ & $\mathrm{CAT} \rightarrow \mathrm{CAC}$ & NO & Not done & This comm. \\
\hline Gly 338Gly (G338G) & $\mathbf{X}$ & $\mathrm{GG} \underline{\mathrm{C}} \rightarrow \mathrm{GG} \underline{\mathrm{G}}$ & YES & Not done & Tyfield, per. comm. \\
\hline
\end{tabular}

${ }^{a} \mathrm{http}: / / \mathrm{www} . c c . e m o r y . e d u / P E D I A T R I C S / m e d g e n /$ research/db.htm

${ }^{b} \mathrm{CpG}$ dinucleotides affected. ${ }^{60}$

' Normal level of GALT in COS cells expression system.

${ }^{d}$ Normal level of GALT in GALT-less yeast and/or GALT-less $E$. coli expression systems.

${ }^{e} \mathrm{http}: / /$ www.ich.bris.ac.uk/galtdb/

$\mathrm{bp}$, base pair; fs, frameshift; del, deletion; $\mathrm{Kb}$, kilobase ; $\mathrm{nt}$, nucleotide; ins, insertion 


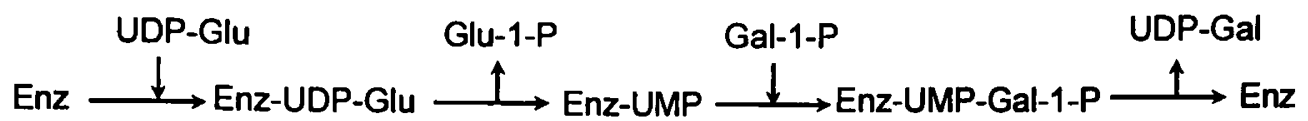

Fig. 2 Galactose 1-phosphate uridyltransferase exhibits ping-pong, bi-bi kinetics. Enz, Enzyme; UDP-Glu, UDP-glucose; Glu-1 P, glucose 1-phosphate; Gal-1-P, galactose 1-phosphate; UDP-Gal, UDP-galactose. See text and reference 61 for explanation.

Little is known about the biochemical mechanism(s) by which glutamine- 188 is essential or how GALT is impaired by the substitution of an arginine for glutamine at this position. By superimposing the human GALT amino acid sequence on the highly homologous three-dimensional crystal structure of the E. coli GALT, 24,25 glutamine-188 lies proximal to and in the beta sheet of $\mathrm{His}^{184}$-Pro ${ }^{185}$-His ${ }^{186}$ which initiates a nucleophilic attack in the release of glucose 1-phosphate from UDP-glucose (Fig. 1). ${ }^{28}$ This short stretch of amino acids is conserved evolutionarily and is crucial for normal catalytic function of $E$. coli GALT.

When human GALT cDNA containing the Q188R mutation was expressed in "knockout" yeast cells, no GALT activity was detected. ${ }^{26}$ These investigators demonstrated that when a Q188R-GALT monomeric subunit dimerized with a wild-type GALT subunit to form a "heterodimer," the resultant catalytic activity of the heterodimer was less than $50 \%$ of when only the wild-type GALT cDNA was expressed. The investigators suggested that the Q188R-GALT subunit exerted a "dominant negative" effect on a wildtype subunit when the two subunits dimerized. ${ }^{29}$ This observation was the first to address potential interactions between the GALT subunits, but did not explain the essentiality of glutamine- 188 or how the Q188R mutation per se inactivated the catalytic function of the mutant enzyme subunits. Others found that patients homozygous for the Q188R mutation had no GALT protein in erythrocytes, leukocytes, or transformed lymphoblasts. ${ }^{30}$ Using site-directed mutagenesis in an E. coli GALT-knockout system, the Q188 residue was found to be crucial for GALT catalysis with normal amounts of protein. ${ }^{27}$ Therefore, the pathobiochemical effects of an arginine substituted for glutamine-188 involve both biostability of the GALT protein and its catalytic function.

The $1135 \mathrm{~L}$ mutation is common among the black population with a prevalence of $62 \%$ of $G$ alleles. ${ }^{32}$ It was classified inaccurately as a neutral polymorphism when expressed in COS cells. ${ }^{15}$ In the GALTknockout yeast system, S135L GALT activity was $5 \%$ of control activity. ${ }^{33}$ Although no GALT activity was detected in erythrocytes or lymphoblasts from patients homozygous for the $\$ 135 \mathrm{~L}$ mutation, some activity was present in leukocytes. ${ }^{30,32}$ Unexpectedly, whole-body oxidation of ${ }^{13} \mathrm{C}$-galactose to ${ }^{13} \mathrm{CO}_{2}$ was normal. ${ }^{32}$ Thus the $\mathrm{S} 135 \mathrm{~L}$ missense mutation is the basis for the "Negro variant" of galactosemia first documented three decades ago. ${ }^{31,34-36}$ One postulated mechanism for the divergence of GALT activity in different organs is that the mutation increases rates of GALT protein degradation in a tissue-specific manner. The requirement of an hydroxyl amino acid at amino acid position 135 may indicate the need for a zinc ion in this loop which is tissue-specific.

Bioinstability of the GALT protein was postulated 20 years ago and is an important component in the pathophysiology of galactosemia. ${ }^{37}$ Missense muta- tions that alter GALT stability include S135L, N314D, $\mathrm{R} 148 \mathrm{~W}$, and H319Q. ${ }^{16,17,32,38}$ Perhaps the most intensively studied mutation that destabilizes the GALT protein is the Duarte allele, N314D.$^{39}$ GALT function is reduced by $50 \%$ in cells from patients what are homozygous for the N314D mutation and has a distinctive shift in its isozyme pattern when analyzed by isoelectric focusing. ${ }^{38,41}$ The bioinstability of the N314D mutation has been quantitated in lymphoblastoid cell lines transformed from patients with these mutations ${ }^{38,39}$ An opposite charge change by a second mutation corrected this effect on biostability. Using lymphoblastoid cells, a "revertant" missense mutation was caused by a lysine substitution for glutamic acid at position 203 (E203K) but only when E203K was in cis with the N314D mutation. This second charge change "rescued" the destabilization effect of the aspartate substitution. ${ }^{38}$ The two mutations lie outside the catalytic region on a loop and an $\alpha$-helix that contains a metal ion requiring hydrogen bonding to ferrous ion in the E. coli protein. ${ }^{24}$

Both the Q188R and N314D were in linkage disequilibrium with a $S a c I$ polymorphism site at nucleotide 1391 in intron $\mathrm{E}$ of the GALT gene. This SacI polymorphism, caused by a $\mathrm{G} \rightarrow \mathrm{A}$ transition, is linked to the Q188R mutation. ${ }^{42}$ The N314D mutation, on the other hand, was found exclusively on chromosomes without the SacI site at nucleotide 1391. It was proposed that the two common mutations, N314D and Q188R, evolved independently on a different chromosomal background. ${ }^{42}$ Different ethnic distributions of missense mutations also support a general theory that mutations causing galactosemia arose after socialization occurred in modern humans (see below).

The Los Angeles (LA) variant of galactosemia consistently yields higher-than-normal levels ( $121 \%)$ of GALT activity in patients' samples. ${ }^{43,44}$ The LA GALT biochemical phenotype is associated with the N314D mutation and has the same characteristic shift in isozyme pattern, but has enzyme activity significantly greater than that expected for N314D alone. ${ }^{43,44}$ By sequencing GALT genes with this biochemical phenotype, a $\mathrm{C} \rightarrow \mathrm{T}$ transition at nucleotide 1721 (L218L) was found consistently in cis with N314D. LA-variant lymphoblasts have increased GALT abundance, but not GALT mRNA or bioinstability. The TTA leucine codon, caused by this transition ( $\underline{C T A} \rightarrow \underline{T T A}$ ), is rare in the GALT gene. Therefore, the $1721 \mathrm{C} \rightarrow \mathrm{T}$ transition may enhance GALT translation through codon preference. ${ }^{44} \mathrm{All}$ of these patients have normal metabolism of galactose and are not at clinical risk.

\section{ETHNIC DISTRIBUTION OF GALT MUTATIONS}

There is a unique distribution of common GALT mutations among different ethnic groups (Table 2 ). The most common classic galactosemic $(G)$ allele is the Q188R mutation ( $\approx 50 \%$ in the galactosemic population). This mutation, although panethnic, 
Table 2

Distribution of mutant alleles by ethnicity

\begin{tabular}{lccccc}
\hline & White & Black & Askenazim Jews & Other ethnicity & Total \\
\hline Q188R & 192 & 12 & 1 & 75 & 280 \\
N314D & 67 & 4 & 0 & 70 & 141 \\
S135L & 0 & 36 & 0 & 4 & 40 \\
L195P & 7 & 0 & 0 & 4 & 11 \\
Y209C & 5 & 0 & 0 & 0 & 5 \\
K285N & 12 & 1 & 0 & 7 & 20 \\
5 kb deletion & 0 & 0 & 7 & 0 & 7 \\
Others & 30 & 14 & 0 & 20 & 64 \\
Total & 313 & 67 & 8 & 180 & 568 \\
\hline
\end{tabular}

is most prevalent among white patients with galactosemia of Western and Northern European ancestry. ${ }^{12,14,20}$ The $\mathrm{K} 285 \mathrm{~N}$ mutation is the second most common mutation among white galactosemic patients and is most prevalent among patients in Southern Germany, Croatia, and Austria. ${ }^{12}$ This missense mutation is not detected by conventional single-strand conformation polymorphism screening; polymerase chain reaction-based restriction analysis or allele specific oligonucleotides hybridization must be used to screen for this common mutation. The $\mathrm{K} 285 \mathrm{~N}$ mutation made up $10 \%$ of the total non-Q188R mutant alleles in one study of a white galactosemic population. Other common mutations found in the white population were Y209C and L195P (Table 2). ${ }^{16}$ Each of these mutations contributed $8.5 \%$ of the non-Q188R mutant allele. The $\mathrm{S} 135 \mathrm{~L}$ mutation, which has a prevalence of $62 \%$ in all $\mathrm{G}$ alleles in our black patient population, ${ }^{31}$ was found exclusively in this ethnic group. The F171S mutation, which was once thought to be linked to S135L, ${ }^{16}$ may also be exclusive to black patients with galactosemia. ${ }^{45}$

Our group identified four "unrelated" Ashkenazim Jewish families whose probands shared the same 5 $\mathrm{kb}$ deletion in the GALT gene. ${ }^{46}$ This mutation produced a naturally occurring knockout cell line which has been useful in exploring alternate pathways for galactose utilization. ${ }^{46}$

Although galactosemia is relatively rare in the Asian populations, ${ }^{47} 12$ mutations were identified in East Asian patients with galactosemia (Japanese).$^{48}$ Four of them, $\Delta \mathrm{C}$ (bp2756), $\Delta \mathrm{T}$ (bp2359), R333W, and P325L, were also identified in the American galactosemic population. Four mutations, ins G528, V150L, P183T, and H319Q, were found exclusively in Italian patients. ${ }^{49}$ The Y323D mutation was found in one Pakistani family.

The Duarte variant (N314D) is found in all populations with a prevalence ranging from $6 \%$ to $20 \%$ of "normal" GALT alleles. ${ }^{50}$ Although there are 131 unique changes found in the GALT gene, many of

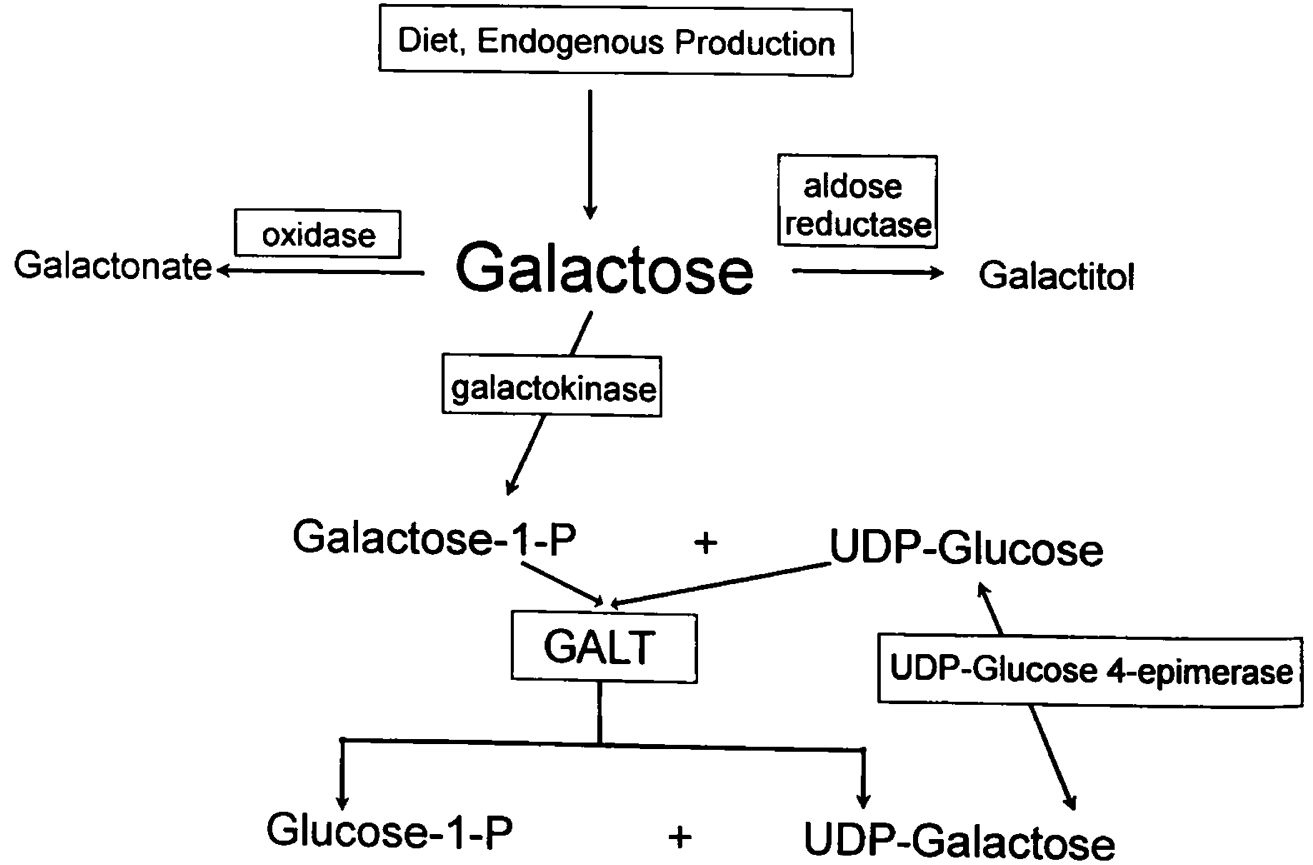

Fg. 1 Metabolic pathways for galactose metabolism. 
these changes are private mutations (with a frequency of less than $0.5 \%$ of $\mathrm{G}$ alleles). It is important to note that six mutations (Q188R, S135L, N314D, K285N, L195P, Y209C) account for $87.5 \%$ of the mutant alleles in our study of 568 mutations associated with galactosemia (Table 2). Since relatively few mutations account for the majority of galactosemic alleles, DNA analysis as a "second tier" to population-based newborn screening for galactosemia and heterozygote detection is feasible for public health programs.

\section{GENOTYPE/PHENOTYPE CORRELATION OF GALT MUTATIONS}

Reports on clinical outcome of genotyped patients with galactosemia are relatively scarce. The small sample size of investigations and the different criteria used for assessment of outcome has produced conflicting observations. We previously reported the association of poor patient outcome and homozygosity for the Q188R mutation and the association of good outcome with homozygosity for the S135L mutation..$^{20,27}$ Others have not found an effect on outcome of the Q188R/Q188R genotype..$^{51}$ Based on patients' residual red cell GALT activity and the rate of decline of galactose 1-phosphate after the initiation of dietary treatment, three patients with the genotypes Q188R/T138M, Q188R/R259W, and Q188R/T350A, respectively, manifested milder clinical symptoms in the newborn period than patients who were homozygous for R333W, Q188R, or L195P. ${ }^{52}$ A good outcome has been associated with the mutations $\mathrm{A} 330 \mathrm{~V}$ and $\mathrm{W} 316 \mathrm{X}$ in two patients. ${ }^{53}$ Patients with GALT genotypes Q212X/Q188R had poor outcomes. ${ }^{54} \mathrm{~A}$ patient who is a compound heterozygote for Q188R/E304X with the LA variant is doing well..$^{55}$ Poor outcome was described in a G/G patient with a genotype of P183T/unknown. ${ }^{49}$ Despite newborn detection and intervention, a teenager homozygous for the $5 \mathrm{~kb}$ deletion also had neurological and ovarian problems. In contrast to earlier proposals, these observations indicate that complete GALT deficiency is compatible with a livebirth and mild long-term complications. ${ }^{19}$

The pathogenesis of chronic complications in galactosemia is complex and remains largely unknown. The severity of incurred neonatal problems is probably the most important variable on outcome. Outcome involves not only the genotype and impairment of GALT, but also epigenetic factors such as the affinity and capacity of other enzymes involved in galactose metabolism (galactose reductase, galactose oxidase, UDP-galactose-4-epimerase, galactokinase). Endogenous galactose production ${ }^{55}$ and intake of galactose are two other important factors for consideration (Fig. 2). It is probable that accumulation of both galactitol and galactose 1-phosphate within cells are combined causes of cellular and organ failure. Decreased availability of UDP-galactose may also result in reduced production of glycolipids and glycoproteins (Fig. 2). A knockout mouse model has helped in understanding this pathophysiology although rodent models lack aldose reductase. ${ }^{56}$ The knockout mouse is homozygous for GALT deficiency, it does not get "sick" and females have normal fertility. Because the mouse has less galactose reductase than primates, it produces less galactitol. Mouse milk contains only $2 \%$ lactose, compared with $6 \%$ in human milk, which represents an additional dimension and emphasizes environmen- tal effects on outcome. Reduced intake of lactose may explain the relatively smaller accumulation of erythrocyte galactose 1-phosphate compared with human newborns with galactosemia. Therefore, environment. genotypic changes in GALT, and epigenetic pathways or galactose metabolism all contribute to outcome in galactosemia.

\section{Acknowledgments}

This research is supported in part by a US Health and Human Services Grant from the National Institute of Child Health and Human Development Grant PO 1 HD29847 01, and by a US Public Health Services Grant M01 RR00039 for the General Clinical Research Center of Emory University and by the N. Rochat Research Fund.

We thank SD Langley, Dr. PP Dembure, Dr. K Muralidharan, and Nick Hjelm for their assistance in biochemical and molecular genotyping. We also thank the many physicians who sent patient samples for biochemical phenotyping and molecular genotyping. We are grateful to Dr. I Raymont and Dr. P Frey for allowing us access to the coordinates of their E. coli GALT crystals.

\section{References}

1. Mason HH, Turner ME. Chronic galactosemia. Am J Dis Child 1935;50:359.

2. Komrower GM. Galactosemia: Thirty years on the experience of a generation. J Inher Metab Dis 1982;5:96-104.

3. Waggoner DD, Buist NR, Donnell GN. Long-term prognosis in galactosemia: Results of a survey of 350 cases. J Inherit Metab Dis 1990;13:802-818.

4. Waggoner DD, Buist NR. Long-term complications in treated galactosemia-175 US cases. Int Pediatr 1993;8:97-100.

5. Segal S, Berry GT. Disorders of galactose metabolism. In: Scriver D, Beaudet A, Sly W, Valle D, editors. The Metabolic Basis of Inherited Disease. New York: McGraw-Hill, 1995:967-1000.

6. Steinmann B, Gitzelmann R, Zachmann M. Hypogonadism and galactosemia [letter]. $N$ Engl J Med 1981;305:464-465.

7. Nelson CD, Waggoner DD, Donnell GN, Tuerch JM, Buist NR. Verbal dyspraxia in treated galactosemia. Pediatrics 1990;88:346-350.

8. Fishler K, Koch R, Donnell GN, Wenz E. Developmental aspects of galactosemia from infancy to childhood. Clin Pediatr 1980;19:38-44.

9. Friedman JH, Levy HL, Boustany RM. Late onset neurologic syndromes in galactosemic siblings. Neurology 1989;39:741-742.

10. Flach JE, Reichardt JK, Elsas LJ. Sequence of a CDNA encoding human galactose-1 phosphate uridyltransferase. Mol Biol Med 1990;7:365-369.

11. Lemaire HG, Mueller-Hill B. Nucleotide sequence of the GalE gene and the GalT gene for E. coli. Nucleic Acids Res 1986;14:7705-7011.

12. Leslie ND, Immerman EB, Flach JE, Florez M, Fridovich-Keil IL, Elsas LJ. The human galactose-1-phosphate uridyltransferase gene. Genomics 1992;14:474-480.

13. Reichardt JK, Berg P. Cloning and characterization of a CDNA encoding human galactose-1-phosphate uridyltransferase. Mol Biol Med 1988;5:107-122.

14. Reichardt JK, Packman S, Woo SLC. Molecular characterization of two galactosemia mutations: Correlation of mutations with highly conserved domains in galactose-1-phosphate uridyltransferase. Am J Hum Genet 1991;49:860-867.

15. Reichardt JK, Woo SLC. Molecular basis of galactosemia: Mutations and polymorphisms in the gene encoding human galactose-1-phosphate uridyltransferase. Proc Natl Acad Sci USA 1991;88:2633-2637.

16. Reichardt JK, Belmont JW, Levy HL, Woo SLC. Characterization of two missense mutations in human galactose-1-phosphate uridyltransferase: Different molecular mechanisms for galactosemia. Genomics 1992;12:596-600.

17. Reichardt JK, Levy HL, Woo SLC. Molecular characterization of two galactosemia mutations and one polymorphism: Impli- 
cations for structure-function analysis of human galactose-1phosphate uridyltransferase. Biochemistry 1992;31:5430-5433.

18. Reichardt JK, Novelli G, Dallapiccola B. Molecular characterization of the H319Q galactosemia mutation. Hum Mol Genet 1993;2:325-326.

19. Reichardt JK. The molecular genetic basis of galactosemia. Int Pediatr 1993;8:110-113.

20. Elsas L], Fridovich-Keil JL, Leslie ND. Galactosemia: A molecular approach to the enigma. Int Pediatr 1993;8:101.

21. Arabshahi A, Brody RS, Smallwood A, Tsai TC, Frey PA. Galactose-1-phosphate uridyltransferase. Purification of the enzyme and stereochemical course of each step of the double displacement mechanism. Biochemistry 1986;25:5583-5589.

22. Markus HB, Wu JW, Boches FS, Tedesco TA, Mellman WI, Klalen RG. Human erythrocyte galactose-1-phosphate uridyltransferase. J Biol Chem 1977;252:5363-5369.

23. Wong LJ, Frey PA. Galactose-1-phosphate uridyltransferase: Isolation of a uridyl-enzyme intermediate. $J$ Biol Chem 1974;249:2322-2324.

24. Wedekind JE, Frey PA, Rayment I. The dimensional structure of galactose-1 phosphate uridyltransferase from Escherichia coli at 1.8 ( resolution. Biochemistry 1995;34:11049-11061.

25. Wedekind JE, Frey PA, Rayment I. The structure of nucleotidylated histidine-166 of galactose-1-phosphate uridyltransferase provides insight into phosphoryl group transfer. Biochemistry 1995:35:11560-11569.

26. Fridovich-Keil JL, Jinks-Robertson S. A yeast expression system for human galactose-1-phosphate uridyltransferase. Proc Natl Acad Sci USA 1993;90:398-402.

27. Lai K, Willis A, Elsas L. Catalytic role of glutamine 188 (Q188) in galactose-1-phosphate uridyltransferase. Am J Hum Genet 1997;61:A255.

28. Field TL, Reznikoff WS, Frey PA. Galactose-1-phosphate uridyltransferase: Identification of histidine-164 and histidine-166 as critical residues by site-directed mutagenesis. Biochemistry 1989;28:2094-2099.

29. Elsevier JP, Fridovick-Keil JL. The Q188R mutation in human galactose-1-phosphate uridyltransferase acts as a partial dominant negative. J Biol Chem 1996;271:32002-32007.

30. Landt M, Ritter D, Lai K, Benke P, Elsas L), Steiner R. Black children deficient in galactose-1-phosphate uridyltransferase: Correlation of activity and immunoreactive protein in erythrocytes and leukocytes. J Pediatr 1997;130:972-980.

31. Segal S, Rogers S, Holtzapple PG. Liver galactose-1-phosphate uridyltransferase: Activity in normal and galactosemic subjects. J Clin Invest 1971;50:500-506.

32. Lai K, Langley SD, Singh R, Hjelm N, Dembure PP, Elsas LJ. A prevalent mutation for galactosemia among black Americans. J Pediatr 1996;128:89-95.

33. Fridovich-Keil JL, Langley SD, Mazur LA, Lennon JC, Dembure PP, Elsas LJ. Identification and functional analysis of three distinct mutations in the human galactose-1-phosphate uridyltransferase gene associated with galactosemia in a single family. Am J Hum Genet 1995;56:640-646.

34. Baker L, Mellman WJ, Tedesco TA, Segal S. Galactosemia: Symptomatic and asymptomatic homozygotes in one Negro sibship. J Pediatr 1966;68:551-558.

35. Segal S, Blair A, Roth $H$. The metabolism of galactose by patients with congenital galactosemia. Am J Med 1965;38:62-70.

36. Segal $\mathrm{S}$, Blair A, Topper YJ. Oxidation of $\mathrm{C}^{14}$ galactose by patients with congenital galactosemia. Science 1962;136:150-151.

37. Chacko CM, Christian JC, Nadler HL. Unstable galactose-1phosphate uridyltransferase: A new variant of galactosemia. I Pediatr 1971;78:454-460.

38. Lai K, Langley SD, Dembure PP, Hjelm N, Elsas LJ. Duarte allele impairs biostability of human galactose-1-phosphate uridyltransferase in human lymphoblasts. Hum Mutat 1998;11:28-38.

39. Elsas LJ, Dembure P, Langley S, Paulk EM, Hjelm LN, FridovichKeil JL. A common mutation associated with the Duarte galactosemia allele. Am J Hum Genet 1994;54:1030-1036.

40. Beutler E, Baluda MC, Sturgeon P, Day R. A new genetic abnormality resulting in galactose-1-phosphate uridyltransferase deficiency. Lancet 1965;1:353-354.

41. Mathai CK, Beutler E. Electrophoretic variation of galactose- 1-phosphate uridyltransferase. Science 1966;154:1179-1180.

42. Lin HC, Reichardt JK. Linkage disequilibrium between a SacI restriction fragment length polymorphism and two galactosemia mutations. Hum Genet 1995;95:353-355.

43. Andersen MW, Williams VP, Sparkes MC, Sparkes RS. Transferase-deficiency galactosemia: Immunochemical studies of the Duarte and Los Angeles variants. Hum Genet 1984;65:287-290.

44. Langley SD, Lai K, Dembure PP, Hjelm N, Elsas LJ. Molecular basis for Duarte and Los Angeles variant galactosemia. Am J Hum Genet 1997;60:366-372.

45. Wang BT, Xu WG, Wong LJ, Kaufman FR, Williams III J, Ng WG. Molecular and biochemical studies in African-American galactosemia patients. Am J Hum Genet 1996;59:A292.

46. Muralidharan $K$, Zhang W, Dembure $P$, Langley $S D$, Lai $K$, Rea $C$, et al. A naturally occurring "knockout" mutation of human galactose-1-phosphate uridyltransferase gene reveals an alternate pathway for galactose metabolism. Am J Hum Genet 1996;59:A204.

47. Ashino I, Okano $Y$, Suyama I, Yamazaki T, Yoshino $M$, Furuyama JI, et al. Molecular characterization of galactosemia (type I) mutations in Japanese. Hum Mutat 1995;6:36-43.

48. Hirokawa H, Okano Y, Ashino J, Imamura T, Suyama I, Isshiki G. Molecular characterization of galactosemia mutations (type I) in Japanese. Presented at the Seventh International Conference on Inborn Error of Metabolism. Vienna, Austria, 1997.

49. Maceratesi $P$, Sangiuolo F, Novelli G, Ninfali $P$, Magnani $M$, Reichardt JK, et al. Three new mutations (P183T, V159L, 528 ins $\mathrm{G}$ ) and eleven sequence polymorphisms in Italian patients with galactose-1-phosphate uridyltransferase (GALT) deficiency. Hum Mutat 1996;8:369-372.

50. Mellman WI, Tedesco TA, Feigl P. Estimation of the gene frequency of the Duarte variant of galactose-1-phosphate uridyltransferase. Am J Hum Genet 1968;32:1-8.

51. Kaufman FR, Xu YK, Ng WG, Donnell GN. Correlation of ovarian function with galactose-1-phosphate uridyltransferase levels in galactosemia. J Pediatr 1988;112:754-6.

52. Shin YS, Gathof BS, Podskarbi, Sommer M, Giugliani R, Gresser U. Three missense mutations in the galactose-1-phosphate uridyltransferase gene of three families with mild galactosemia. Eur J Pediatr 1996;133:393-397.

53. Sommer M, Gathof BS, Podskarbi T, Giugliani R, Kleinlein B, Shin YS. Mutations in the galactose-1-phosphate uridyltransferase gene of two families with mild galactosemia variants. J Inherit Metab Dis 1995;18:567-576.

54. Gathof BS, Sommer M, Podskarbi T, Reichardt J, Braun A, Gresser $U$, et al. Characterization of two stop codon mutations in the galactose-1-phosphate uridyltransferase gene of three male galactosemic patients with severe clinical manifestation. Hum Genet 1995;96:721-725.

55. Berry GT, Nissim I, Gibson JB, Masur AT, Lin Z, Elsas LJ, et al. Quantitative assessment of whole body galactose metabolism in galactosemic patients. Eur I Pediatr Suppl 1997;56:S43-S49.

56. Leslie ND, Yager KI, McNamara PD, Segal S. A mouse model galactose-1-phosphate uridyitransferase deficiency. Biochem Mol Med 1996;59:7-12.

57. Ito M,Amet $\mathrm{N}$, Chen $\mathrm{T}$, Wong LJC. Detection of novel mutations in galactose-1-phosphate uridyltransferase (GALT) gene in galactosemia patients by temporal temperature gradient gel electrophoresis (TGGE). Am J Hum Genet 1997;61:A252.

58. Elsas LJ, Langley SD, Steele E, Evinger J, Fridovich-Keil Л, Brown A, et al. Galactosemia: A strategy to identify new biochemical phenotypes and molecular genotypes. Am J Hum Genet 1995;56:630-639.

59. Wadelius C, Lagerkvist A, Molin AK, Larsson A, von Dobeln $U$, Pettersson U. Galactosemia caused by a point mutation that activates cryptic donor splice site in the galactose-1phosphate uridyltransferase gene. Genomics 1993;17:525-526.

60. Cooper DN, Youssonffian H. The CpG dinucleotides and human genetic diseases. Hum Mutat 1988;78:151-155.

61. Frey PA. Nucleotidyltransferases and phosphotransferases: Stereochemistry and covalent intermediates. The Enzymes 1992;XX:141-186. 